\title{
Hemodinâmica de diferentes frações inspiradas de oxigênio em cães submetidos à infusão contínua de propofol sob ventilação espontânea
}

\author{
Hemodyinamic effects of several inspired oxygen fractions in spontaneously breathing \\ dogs submitted to continuous infusion of propofol \\ Newton Nunes $^{{ }^{*}}$ Patrícia Cristina Ferro Lopes ${ }^{\mathrm{II}}$ Paulo Sérgio Patto dos Santos III \\ Danielli Parrilha de PaulaII Celina Tie Duque NishimoriII Roberta Carareto ${ }^{\text {II }}$ \\ Marlos Gonçalves SousaII Vivian Fernanda Barbosa ${ }^{\text {II }}$ \\ Aparecido Antonio Camacho ${ }^{\text {II }}$
}

RESUMO

Avaliaram-se os efeitos do fornecimento de diferentes frações inspiradas de oxigênio ( $\left.\mathrm{FiO}_{2}\right)$ sobre os parâmetros hemodinâmicos em cães submetidos à infusão contínua de propofol e mantidos em ventilação espontânea. Foram utilizados oito cães, os quais foram empregados em cinco grupos com diferentes $\mathrm{FiO}_{2}$, G100 $\left(\mathrm{FiO}_{2}=1\right), \mathrm{G80}\left(\mathrm{FiO}_{2}=0,8\right)$, $G 60\left(\mathrm{FiO}_{2}=0,6\right), \mathrm{G} 40\left(\mathrm{FiO}_{2}=0,4\right)$ e $\mathrm{G} 20\left(\mathrm{FiO}_{2}=0,21\right)$, respeitando-se um intervalo de dez dias entre cada procedimento anestésico. Os animais foram induzidos e mantidos sob anestesia com propofol na dose de $0,7 \mathrm{mg} \mathrm{kg}^{-1} \mathrm{~min}^{-1}$ e, após a intubação orotraqueal, iniciou-se o fornecimento de oxigênio conforme a $\mathrm{FiO}_{2}$ determinada para cada grupo. As primeiras mensurações, da freqüência cardíaca $(F C)$, das pressões arteriais sistólica, diastólica e média (PAS, PAD e PAM), da resistência vascular periférica e pulmonar (RPT e RPV), do débito cardíaco (DC); da pressão venosa central (PVC), da pressão média da artéria pulmonar (PMAP), da pressão média capilar pulmonar (PMCP), da pressão parcial de oxigênio $\left(\mathrm{PaO}_{2}\right)$ e do dióxido de carbono $\left(\mathrm{PaCO}_{2}\right)$ no sangue arterial, foram efetuadas 30 minutos após o início da infusão do anestésico (M0), seguidas de intervalos de 15 minutos (M15, M30, M45 e M60). Os dados numéricos obtidos foram submetidos à Análise de Variância, seguida pelo teste Tukey $(P<0,05)$. Os parâmetros hemodinâmicos não apresentaram diferenças significativas ao longo do tempo e entre os grupos. Os resultados obtidos permitiram concluir que as variáveis hemodinâmicas não são afetadas pelo emprego de diferentes $\mathrm{FiO}_{2}$.

Palavras-chave: frações inspiradas de oxigênio, propofol, cão, parâmetros hemodinâmicos.

\section{ABSTRACT}

The effects of several inspired oxygen fractions $\left(\mathrm{FiO}_{2}\right)$ in hemodynamics parameters in spontaneously breathing dogs submitted to continuous infusion of propofol were evaluated. Eight adult mongrel dogs were studied and the animals underwent five anesthesias. In each procedure the patient was submitted to a different $\mathrm{FiO}_{2}$, thereby resulting in five groups, namely: $\mathrm{G} 100\left(\mathrm{FiO}_{2}=1\right), \mathrm{G} 80\left(\mathrm{FiO}_{2}=0.8\right), \mathrm{G} 60$ $\left(\mathrm{FiO}_{2}=0.6\right), \mathrm{G} 40\left(\mathrm{FiO}_{2}=0.4\right)$, and $\mathrm{G} 20\left(\mathrm{FiO}_{2}=0.21\right)$. The dose of propofol was sufficient to abolish protector reflex and ensure endotracheal intubation, followed by immediate continuous infusion of that drug $\left(0.7 \mathrm{mg} \mathrm{kg}^{-1} \mathrm{~min}^{-1}\right)$. The initial measurement (MO) of heart rate (HR); systolic (SAP), diastolic (DAP) and mean pressure (MAP), systemic (SVR) and pulmoary vascular resistance (PVR), cardiac output (CO); central venous pressures (CVP); mean pulmonary pressure (MPAP); pulmonary capillary wedge pressure (PCWP), arterial partial pressure of oxygen $\left(\mathrm{PaO}_{2}\right)$ and carbon dioxide $\left(\mathrm{PaCO}_{2}\right)$ were recorded thirty minutes after the beginning of propofol infusion. Additional recordings were performed at each 15-minute interval during 60 minutes (M15, M30, M45, and M60). Numeric data were submitted to Analysis of Variance followed by Tukey Test $(P<0.05)$. Significant values of hemodynamic parameters were not recorded neither during the experiment period nor among the groups. In conclusion, different $\mathrm{FiO}_{2}$ does not impair hemodynamic parameters.

Key words: oxygen inspired fraction, propofol, dog, hemodynamic parameters.

\section{INTRODUÇÃO}

Na Medicina Veterinária, trabalhos empregando o propofol como agente anestésico têm proporcionado embasamento teórico para que seja cada

\footnotetext{
'Departamento de Clínica e Cirurgia Veterinária, Faculdade de Ciências Agrárias e Veterinárias (FCAV), Universidade Estadual Paulista (UNESP). Campus de Jaboticabal, Via de Acesso Prof. Paulo Donato Castellane, s/n, Zona Rural, 14884-900, Jaboticabal, SP, Brasil. E-mail: newton@fcav.unesp.br.*Autor para correspondência.

IIPrograma de Pós-graduação, FCAV/UNESP, Jaboticabal, SP, Brasil.

IIIDepartamento de Clínica, Cirurgia e Reprodução Animal, Faculdade de Medicina Veterinária de Araçatuba, UNESP, Araçatuba, SP, Brasil.
} 
vez mais freqüente seu emprego na rotina anestésica, pois a infusão contínua desse fármaco apresenta-se como uma alternativa importante em substituição à anestesia inalatória, por apresentar indução (DUKE,1995) e recuperação anestésicas rápidas (WHITE, 2001), poucos efeitos adversos e ausência de efeito cumulativo (MORGAN \& LEGGE, 1989).

No entanto, a preocupação com a ventilação e conseqüente oxigenação do paciente, durante os procedimentos anestésicos, é de extrema importância, pois a função primordial da integração dos sistemas cardiovascular e respiratório é suprir as necessidades metabólicas dos diversos tecidos do organismo, por meio da oferta adequada de oxigênio $\left(\mathrm{O}_{2}\right)$ (ROMALDINI, 1995). Entretanto, deve-se ressaltar que prejuízos na troca gasosa durante a anestesia geral podem decorrer da formação de áreas de atelectasia tanto em anestesias inalatórias como em intravenosas (STRANDBERG et al., 1986), sendo que altas frações inspiradas de oxigênio $\left(\mathrm{FiO}_{2}\right)$ durante esses procedimentos freqüentemente estão correlacionadas com tal ocorrência (MAGNUSSON \& SPAHN, 2003). Dessa forma, baixas concentrações desse gás têm sido utilizadas para evitar tal problema (AKÇA et al.,1999; ROTHEN et al., 1996).

Novos estudos têm avaliado o emprego de baixas $\mathrm{FiO}_{2}$ no sistema cardiovascular. FUIJI et al. (2001), utilizando $\mathrm{FiO}_{2}=0,4$ em cães submetidos à infusão de propofol, observaram redução da freqüência cardíaca (FC) e da pressão arterial média (PAM), sendo tal fato atribuído à infusão do fármaco, pois, 10 minutos após o término da sua administração, os valores retornaram a seus patamares iniciais. Em outra pesquisa, ZAPPELLINI et al. (1996), em seu estudo com cães, não registraram diferenças significativas nos parâmetros hemodinâmicos entre os grupos ventilados com $\mathrm{FiO}_{2}=1$ e $\mathrm{FiO}_{2}=0,21$.

Em contrapartida, em estudo com diferentes concentrações de $\mathrm{O}_{2}(11,21$ e 100\%), CLAYBAUGH et al. (2003) afirmaram que o emprego de $\mathrm{FiO}_{2}=1$ durante hemorragia induzida em cabras manteve a pressão sangüínea arterial, melhorando, portanto, a pressão de perfusão dos tecidos. Entretanto, KIM et al. (1998), em seu estudo com ratos submetidos a choque hemorrágico letal, observaram que a $\mathrm{FiO}_{2}=1$ proporcionou aumento da $\mathrm{PaO}_{2}$, mas não alterou a PAM.

Objetivou-se, com este trabalho, determinar os efeitos do emprego de diferentes frações inspiradas de oxigênio nas variáveis cardiovasculares em cães mantidos em ventilação espontânea e submetidos à infusão contínua de propofol.

\section{MATERIAL E MÉTODOS}

Foram utilizados oito cães adultos, machos ou fêmeas, sem raça definida, evitando-se animais prenhes ou em estro, pesando entre 5 e $11 \mathrm{~kg}$, considerados hígidos após exames físico e a complementares, dentre os quais a hematológico, urinálise, o eletrocardiográfico e radiografias torácicas. Os animais foram mantidos em canis individuais.

Cada cão foi submetido a cinco procedimentos com infusão contínua de propofol, permitindo, portanto, a formação de cinco grupos experimentais denominados $\mathrm{G} 100\left(\mathrm{FiO}_{2}=1\right)$, G80 $\left(\mathrm{FiO}_{2}=0,8\right), \mathrm{G} 60\left(\mathrm{FiO}_{2}=0,6\right), \mathrm{G} 40\left(\mathrm{FiO}_{2}=0,4\right)$ e $\mathrm{G} 20$ $\left(\mathrm{FiO}_{2}=0,21\right)$, sendo respeitado um intervalo de dez dias entre cada anestesia. Para o início do estudo, escolheu-se $\mathrm{FiO}_{2}$ a ser empregada ao acaso, iniciandose pela concentração de $\mathrm{O}_{2}$ de $60 \%$, seguida pelas frações inspiradas de 100, 80, 40 e 21\%.

Todos os animais foram induzidos à anestesia geral pela administração intravenosa de propofol $^{\mathrm{a}}$ na dose necessária para perda do reflexo laringotraqueal. Em seguida, os animais foram intubados e a sonda foi conectada ao circuito anestésico com reinalação parcial de gases ${ }^{\mathrm{b}}$, para o fornecimento de $\mathrm{O}_{2}$ conforme as $\mathrm{FiO}_{2}$ determinadas em cada grupo, sendo o fluxo total mantido de $30 \mathrm{a} 50 \mathrm{ml} \mathrm{kg}^{-1} \mathrm{~min}^{-1}$. Iniciou-se então a infusão contínua de propofol, por meio de bomba de infusão ${ }^{c}$, empregando-se a dose de $0,7 \mathrm{mg}$ $\mathrm{kg}^{-1} \mathrm{~min}^{-1}$. A leitura da concentração do gás foi obtida pelo uso de analisador de gases ${ }^{\mathrm{d}}$, cujo sensor se encontrava adaptado na extremidade da sonda orotraqueal conectado ao equipamento de anestesia.

Procedeu-se à cateterização transcutânea da artéria társica ${ }^{\mathrm{e}}$ para mensuração das pressões arteriais sistólica (PAS), diástólica (PAD), média (PAM) e para coleta de sangue para hemogasometria e mensuração da pressão parcial de oxigênio no sangue arterial $\left(\mathrm{PaO}_{2}\right)$, pressão parcial de dióxido de carbono no sangue arterial $\left(\mathrm{PaCO}_{2}\right)$ e $\mathrm{pH}^{\mathrm{f}}$. No terço médio da região cervical, localizou-se a veia jugular esquerda para a introdução do catetere, para posterior introdução de catéter de termodiluiçãog ${ }^{g}$, cuja extremidade distal foi posicionada na luz da artéria pulmonar, observando-se as formas das ondas de pressão, segundo descrito por SISSON (1992), para mensuração do débito cardíaco ${ }^{d}$ (DC), da pressão venosa central (PVC), da pressão média da artéria pulmonar (PMAP) e da pressão média capilar pulmonar (PMCP). A resistência periférica total (RPT) e a resistência vascular pulmonar (RVP) foram obtidas por cálculos matemáticos descritos por VALVERDE et al. (1991) e NUNES (2002).

A FC foi obtida calculando-se o intervalo de tempo constituído entre dois intervalos R-R 
consecutivos do traçado eletrocardiográfico, registrado por método computadorizado ${ }^{\mathrm{h}}$ e ajustado para leitura na derivação II.

As observações das variáveis iniciaram-se após 30 minutos da infusão contínua de propofol (M0). Novas mensurações foram realizadas em intervalos de 15 minutos, por um período de 60 minutos (M15, M30, M45 e M60, respectivamente). Todas as variáveis foram submetidas à Análise de Variância (ANOVA), seguida pelo teste de Tukey $(\mathrm{P}<0,05)$ para avaliação individual dos grupos e entre eles.

\section{RESULTADOS E DISCUSSÃO}

Relativamente à FC, foi possível observar, em todos os grupos, estabilidade das médias (Tabela 1), com valores situados dentro do intervalo considerado fisiológico para a espécie segundo TILLEY (1992), não sendo observadas alterações decorrentes do emprego de diferentes $\mathrm{FiO}_{2}$. QUANDT et al. (1998) também observaram estabilidade desse parâmetro em cães, após 30 minutos de anestesia com propofol recebendo $\mathrm{FiO}_{2}=1$. O mesmo foi observado por FERRO et al. (2005), que não encontraram alterações significativas na FC após a administração de diferentes doses de propofol $(0,2$; 0,4 e $0,8 \mathrm{mg} \mathrm{kg}^{-1} \mathrm{~min}^{-1}$ ) em cães respirando espontaneamente ar ambiente.

A PAS, a PAD e a PAM não apresentaram diferenças entre os momentos em todos os grupos, sendo observados valores significativos entre os grupos apenas em M60, no qual o G20 foi maior que o G100 e G80 para a PAS, enquanto para PAM maior que

Tabela 1 - Valores médios e desvios-padrão $(\mathrm{x} \pm \mathrm{s})$ de FC (batimento minuto $\left.{ }^{-1}\right)$, PAS $(\mathrm{mmHg}), \mathrm{PAD}(\mathrm{mmHg}), \mathrm{PAM}(\mathrm{mmHg})$ e DC $\left(\mathrm{L}\right.$ min $\left.{ }^{-1}\right)$ em cães submetidos à infusão contínua de propofol $\left(0,7 \mathrm{mg} \mathrm{kg}^{-1} \mathrm{~min}^{-1}\right)$, mantidos em ventilação espontânea com diferentes frações inspiradas de oxigênio [100\% (G100), 80\%(G80), 60\% (G60), 40\% (G40) ou 21\% (G20)].

\begin{tabular}{|c|c|c|c|c|c|c|}
\hline \multirow{2}{*}{ Parâmetros } & \multirow{2}{*}{ Grupos } & \multicolumn{5}{|c|}{ Momentos } \\
\hline & & M0 & M15 & M30 & M45 & M60 \\
\hline \multirow{5}{*}{ FC } & G100 & $111 \pm 15,2$ & $108 \pm 13,5$ & $113 \pm 11,1$ & $112 \pm 10,0$ & $120 \pm 16,2$ \\
\hline & G80 & $123 \pm 26,9$ & $123 \pm 15,7$ & $126 \pm 18,2$ & $125 \pm 20,9$ & $116 \pm 17,9$ \\
\hline & G60 & $112 \pm 7,2$ & $108 \pm 10,1$ & $105 \pm 12,1$ & $107 \pm 13,8$ & $106 \pm 11,3$ \\
\hline & G40 & $114 \pm 27,5$ & $115 \pm 24,5$ & $115 \pm 22,5$ & $114 \pm 19,2$ & $121 \pm 18,4$ \\
\hline & G20 & $114 \pm 15,7$ & $115 \pm 28,3$ & $116 \pm 10,3$ & $130 \pm 25,7$ & $125 \pm 18,2$ \\
\hline \multirow{5}{*}{ PAS } & G100 & $124 \pm 31,2$ & $138 \pm 27,3$ & $124 \pm 21,3$ & $133 \pm 31,7$ & $131 \pm 39,1^{\mathrm{A}}$ \\
\hline & G80 & $129 \pm 34,5$ & $121 \pm 32,2$ & $123 \pm 26,0$ & $123 \pm 31,5$ & $126 \pm 35,5^{\mathrm{A}}$ \\
\hline & G60 & $138 \pm 31,4$ & $145 \pm 23,0$ & $147 \pm 38,9$ & $144 \pm 32,0$ & $143 \pm 26,1$ \\
\hline & G40 & $135 \pm 33,9$ & $129 \pm 35,2$ & $139 \pm 35,0$ & $141 \pm 28,1$ & $150 \pm 26,7$ \\
\hline & G20 & $134 \pm 24,0$ & $141 \pm 31,4$ & $136 \pm 29,1$ & $168 \pm 48,6$ & $181 \pm 42,2^{B}$ \\
\hline \multirow{5}{*}{ PAD } & G100 & $69 \pm 20,9$ & $65 \pm 10,9$ & $61 \pm 14,1$ & $70 \pm 14,8$ & $69 \pm 15,0$ \\
\hline & G80 & $66 \pm 24,5$ & $62 \pm 18,7$ & $69 \pm 21,0$ & $63 \pm 17,7$ & $66 \pm 22,7$ \\
\hline & G60 & $70 \pm 16,9$ & $72 \pm 12,1$ & $76 \pm 22,6$ & $74 \pm 15,9$ & $69 \pm 10,8$ \\
\hline & G40 & $73 \pm 22,1$ & $67 \pm 24,5$ & $69 \pm 23,4$ & $69 \pm 19,5$ & $77 \pm 21,7$ \\
\hline & G20 & $69 \pm 17,1$ & $71 \pm 17,6$ & $71 \pm 21,0$ & $92 \pm 26,2$ & $94 \pm 32,4$ \\
\hline \multirow{5}{*}{ PAM } & G100 & $92 \pm 20,1$ & $90 \pm 14,8$ & $85 \pm 14,6$ & $92 \pm 20,2$ & $90 \pm 20,7$ \\
\hline & G80 & $88 \pm 30,5$ & $81 \pm 22,7$ & $84 \pm 22,8$ & $84 \pm 22,1$ & $83 \pm 21,2^{\mathrm{A}}$ \\
\hline & G60 & $93 \pm 19,4$ & $96 \pm 14,7$ & $100 \pm 27,2$ & $99 \pm 18,1$ & $94 \pm 13,2$ \\
\hline & G40 & $94 \pm 26,2$ & $87 \pm 28,9$ & $91 \pm 25,8$ & $93 \pm 22,2$ & $100 \pm 23,3$ \\
\hline & G20 & $90 \pm 19,5$ & $95 \pm 22,2$ & $93 \pm 22,2$ & $117 \pm 31,8$ & $112 \pm 37,1^{\mathrm{B}}$ \\
\hline \multirow{5}{*}{ DC } & G100 & $1,7 \pm 0,4$ & $1,6 \pm 0,4$ & $1,7 \pm 0,5$ & $1,7 \pm 0,4$ & $1,8 \pm 0,4$ \\
\hline & G80 & $1,8 \pm 0,7$ & $1,7 \pm 0,7$ & $1,8 \pm 0,6$ & $1,8 \pm 0,6$ & $1,9 \pm 0,4$ \\
\hline & G60 & $1,9 \pm 0,7$ & $1,8 \pm 0,5$ & $2,0 \pm 0,5$ & $1,9 \pm 0,6$ & $1,8 \pm 0,5$ \\
\hline & G40 & $1,8 \pm 0,5$ & $1,8 \pm 0,5$ & $1,8 \pm 0,5$ & $1,8 \pm 0,5$ & $2,1 \pm 0,6$ \\
\hline & G20 & $1,7 \pm 0,3$ & $1,9 \pm 0,2$ & $2,0 \pm 0,4$ & $2,1 \pm 0,4$ & $2,1 \pm 0,5$ \\
\hline
\end{tabular}

Médias seguidas por letras maiúsculas diferentes, nas colunas, diferem entre si pelo teste de Tukey $(\mathrm{P}<0,05)$. Médias seguidas por letras minúsculas diferentes, nas linhas, diferem entre si pelo teste de Tukey $(\mathrm{P}<0,05)$.

Ciência Rural, v.38, n.3, mai-jun, 2008. 
G80. Apesar de a PAD não apresentar diferença estatística entre os grupos, a média do G20 também apresentou valores absolutos superiores aos de G80 e G100 no M60 (Tabela 1).

De acordo com GUYTON (1992), os quimiorreceptores são células quimiossensíveis à falta de $\mathrm{O}_{2}$ e ao excesso de dióxido de carbono $\left(\mathrm{CO}_{2}\right)$ ou de íons de hidrogênio, sendo sua localização em vários pequenos órgãos, dois corpos carotídeos e vários corpos aórticos. Os sinais transmitidos desses receptores para o centro vasomotor proporcionam, nessas condições, aumento da pressão arterial.

Dessa forma, sugere-se que os quimiorreceptores tenham sido ativados, não permitindo, portanto, reduções nas pressões arteriais em nenhum grupo, conforme descrito por BUFALARI et al. (1996) e FERRO et al. (2005), uma vez que, em todos os grupos, foi observado excesso de dióxido de carbono e de íons de hidrogênio, conforme demonstrado pelos valores de $\mathrm{PaCO}_{2}$ e pH (Tabela 2). Além disso, os valores registrados para estes parâmetros foram maiores e menores, respectivamente, aos considerados fisiológicos (HASKINS, 1996; SENIOR, 1997), e adicionalmente, em G20, registrou-se baixa tensão de oxigênio.

Quanto às diferenças entre os grupos registradas nas PAS e PAM, essas confirmam a hipótese anteriormente mencionada, uma vez que, no G20, a média de $\mathrm{PaO}_{2}$ foi inferior a 90mmHg (Tabela 2). Como a estimulação dos quimiorreceptores pode ser gerada a partir de tensões de $\mathrm{CO}_{2}$ acima de $30 \mathrm{mmHg}$ e de tensões de $\mathrm{O}_{2}$ menores ou iguais a $90 \mathrm{mmHg}$ (DETWEILER, 1993), no G20, houve um fator a mais de estimulação, proporcionando valores de pressão superiores aos grupos G100 e G80, que, em contrapartida, apresentaram valores significativamente maiores de $\mathrm{PaO}_{2}$. Em cães anestesiados, são previstos valores de $\mathrm{PaO}_{2}$ aproximadamente quatro a cinco vezes maiores à $\mathrm{FiO}_{2}$ fornecida, corroborando os valores registrados neste estudo. Dessa forma, as diferenças registradas podem ser justificadas pelo emprego de diferentes $\mathrm{FiO}_{2}$ em cada grupo (Tabela 2). No G20, a $\mathrm{PaO}_{2}$ no $\mathrm{M} 0$ e no M15 apresentou-se inferior aos valores considerados fisiológicos (HASKINS, 1996), o que pode ter ocorrido pela baixa tensão de $\mathrm{O}_{2}$ fornecida. Do mesmo modo, WATKINS et al. (1987), após a indução anestésica com propofol, observaram $\mathrm{PaO}_{2}$ igual a $77 \mathrm{mmHg}$ em cães saudáveis respirando ar ambiente.

Em relação aos valores de $\mathrm{PaCO}_{2}$, como citado anteriormente, todos os grupos apresentaram valores acima dos fisiológicos. Todavia, o grupo que recebeu oxigênio a 21\% registrou média abaixo de

Tabela 2 - Valores médios e desvios-padrão $(\mathrm{x}+\mathrm{s})$ de $\mathrm{PaO}_{2}(\mathrm{mmHg}), \mathrm{PaCO}_{2},(\mathrm{mmHg})$ e pH em cães submetidos à infusão contínua de propofol $\left(0,7 \mathrm{mg} \mathrm{kg}^{-1} \mathrm{~min}^{-1}\right)$, mantidos em ventilação espontânea com diferentes frações inspiradas de oxigênio [100\% (G100), 80\%(G80), $60 \%$ (G60), 40\% (G40) ou 21\% (G20)].

\begin{tabular}{|c|c|c|c|c|c|c|}
\hline & & \multicolumn{5}{|c|}{ Momentos } \\
\hline & & M0 & M15 & M30 & M45 & M60 \\
\hline \multirow{5}{*}{$\mathrm{PaO}_{2}$} & G100 & $462 \pm 129,3^{\mathrm{A}}$ & $434 \pm 62,8^{\mathrm{A}}$ & $424 \pm 127,6^{\mathrm{A}}$ & $434 \pm 95,9^{\mathrm{A}}$ & $446 \pm 76,1^{\mathrm{A}}$ \\
\hline & G80 & $365 \pm 79,8^{\mathrm{A}}$ & $395 \pm 60,2^{\mathrm{A}}$ & $405 \pm 54,2^{\mathrm{A}}$ & $384 \pm 88,0^{\mathrm{A}}$ & $357 \pm 34,3^{\mathrm{AB}}$ \\
\hline & G60 & $240 \pm 65,4^{\mathrm{B}}$ & $293 \pm 43,1^{\mathrm{B}}$ & $259 \pm 68,8^{\mathrm{B}}$ & $235 \pm 64,2^{\mathrm{B}}$ & $275 \pm 0,7^{\mathrm{BC}}$ \\
\hline & G40 & $179 \pm 16,2^{\mathrm{BC}}$ & $167 \pm 23,2^{\mathrm{C}}$ & $169 \pm 28,0^{\mathrm{BC}}$ & $175 \pm 39,0^{\mathrm{BC}}$ & $181 \pm 22,0^{C D}$ \\
\hline & G20 & $74 \pm 22,0^{\mathrm{C}}$ & $79 \pm 18,4^{\mathrm{D}}$ & $87 \pm 19,9^{\mathrm{C}}$ & $85 \pm 24,2^{\mathrm{C}}$ & $81 \pm 21,3^{\mathrm{D}}$ \\
\hline \multirow{5}{*}{$\mathrm{PaCO}_{2}$} & G100 & $70 \pm 29,5$ & $78 \pm 28,2$ & $96 \pm 39,0^{\mathrm{A}}$ & $91 \pm 41,3$ & $90 \pm 37,2$ \\
\hline & G80 & $73 \pm 27,4$ & $79 \pm 30,9$ & $74 \pm 20,4$ & $88 \pm 31,6$ & $98 \pm 52,5$ \\
\hline & G60 & $68 \pm 18,9$ & $67 \pm 18,5$ & $73 \pm 23,4$ & $74 \pm 22,7$ & $77 \pm 27,0$ \\
\hline & G40 & $53 \pm 11,2$ & $60 \pm 15,6$ & $62 \pm 20,5$ & $69 \pm 22,1$ & $67 \pm 18,8$ \\
\hline & G20 & $53 \pm 11,1$ & $54 \pm 9,8$ & $54 \pm 10,1^{\text {B }}$ & $55 \pm 12,2$ & $57 \pm 11,8$ \\
\hline \multirow{5}{*}{$\mathrm{pH}$} & G100 & $7,207 \pm 0,2$ & $7,157 \pm 0,1$ & $7,097 \pm 0,2$ & $7,112 \pm 0,2$ & $7,107 \pm 0,1$ \\
\hline & G80 & $7,170 \pm 0,1$ & $7,146 \pm 0,1$ & $7,157 \pm 0,1$ & $7,106 \pm 0,1$ & $7,076 \pm 0,2$ \\
\hline & G60 & $7,204 \pm 0,1$ & $7,220 \pm 0,1$ & $7,187 \pm 0,1$ & $7,183 \pm 0,1$ & $7,173 \pm 0,1$ \\
\hline & G40 & $7,256 \pm 0,1$ & $7,216 \pm 0,1$ & $7,193 \pm 0,1$ & $7,177 \pm 0,1$ & $7,187 \pm 0,1$ \\
\hline & G20 & $7,284 \pm 0,1$ & $7,265 \pm 0,1$ & $7,262 \pm 0,1$ & $7,257 \pm 0,1$ & $7,238 \pm 0,1$ \\
\hline
\end{tabular}

Médias seguidas por letras maiúsculas diferentes, nas colunas, diferem entre si pelo teste de Tukey $(\mathrm{P}<0,05)$. Médias seguidas por letras minúsculas diferentes, nas linhas, diferem entre si pelo teste de Tukey $(\mathrm{P}<0,05)$.

Ciência Rural, v.38, n.3, mai-jun, 2008. 
60mmHg. No entanto, somente em M30 foi registrada média de G100 maior que a de G20 (Tabela 2). Observando-se os valores absolutos dessa variável, foi possível detectar o aumento das médias, seguido pelas crescentes $\mathrm{FiO}_{2}$ fornecidas, sendo que quadros de hipercapnia, observados nesse estudo, podem ter sido causados por atelectasia(O’FLAHERTY,1994). Esta proposta pode ser embasada ainda pelo emprego de $\mathrm{FiO}_{2}=1,0$ ou 0,8 (JEFFERIES, 1994), justificando as diferenças nas médias de $\mathrm{PaCO}_{2}$, já que a formação de áreas de colapso pulmonar compromete a troca gasosa e, conseqüentemente, a liberação de $\mathrm{CO}_{2}$.

No pH, não houve diferenças entre os grupos e, dentro destes, entre os momentos (Tabela 2). Apesar de terem sido registradas diferenças nos valores de $\mathrm{PaCO}_{2}$ e de maiores médias desse parâmetro proporcionrem aumento de ácido carbônico (HOUPT, 1993) e redução de pH (MUIR III, HUBBELL, 1997), acredita-se que a ocorrência de reações tampões, bem como de mecanismos compensatórios, possam ter evitado a ocorrência de diferenças entre os grupos.

$\mathrm{O}$ emprego de diferentes $\mathrm{FiO}_{2}$ não permitiu o registro de diferenças significativas na PVC entre os momentos e grupos, sendo que as médias registradas foram consideradas normais para a espécie, segundo HASKINS (1996) (Tabela 3). Os resultados obtidos corroboram os achados de FUJII et al. (2001), que também registraram valores normais em cães sob ventilação mecânica com $\mathrm{FiO}_{2}=0,4$, tratados ou não com propofol.

A PMAP é muito menor que a sistêmica, sendo da ordem de $15 \mathrm{mmHg}$ nos mamíferos

Tabela 3 - Valores médios e desvios-padrão $(\mathrm{x} \pm \mathrm{s})$ de PVC (mmHg), PAPm (mmHg), PCPm (mmHg), RPT (dinaxseg cm ${ }^{-5}$ ) e RVP (dinaxseg $\left.\mathrm{cm}^{-5}\right)$ em cães submetidos à infusão contínua de propofol $\left(0,7 \mathrm{mg} \mathrm{kg}^{-1} \mathrm{~min}^{-1}\right)$, mantidos em ventilação espontânea com diferentes frações inspiradas de oxigênio [100\% (G100), 80\%(G80), 60\% (G60), 40\% (G40) ou 21\% (G20)].

\begin{tabular}{|c|c|c|c|c|c|c|}
\hline \multirow{2}{*}{ Parâmetros } & \multirow{2}{*}{ Grupos } & \multicolumn{5}{|c|}{ Momentos } \\
\hline & & M0 & M15 & M30 & M45 & M60 \\
\hline \multirow{5}{*}{ PVC } & G100 & $7 \pm 7,8$ & $5 \pm 8,1$ & $5 \pm 8,7$ & $6 \pm 9,6$ & $6 \pm 8,2$ \\
\hline & G80 & $5 \pm 4,5$ & $6 \pm 4,2$ & $5 \pm 3,7$ & $5 \pm 3,5$ & $8 \pm 6,7$ \\
\hline & G60 & $4 \pm 3,0$ & $2 \pm 1,3$ & $3 \pm 1,8$ & $6 \pm 2,7$ & $6 \pm 2,7$ \\
\hline & G40 & $4 \pm 3,7$ & $5 \pm 3,0$ & $5 \pm 3,5$ & $5 \pm 3,5$ & $3 \pm 2,4$ \\
\hline & G20 & $4 \pm 2,1$ & $3 \pm 2,8$ & $5 \pm 2,6$ & $5 \pm 4,8$ & $6 \pm 5,6$ \\
\hline \multirow{5}{*}{ PMAP } & G100 & $15 \pm 4,4$ & $14 \pm 4,8$ & $14 \pm 5,6$ & $15 \pm 7,5$ & $17 \pm 6,0$ \\
\hline & G80 & $16 \pm 4,6$ & $17 \pm 5,4$ & $16 \pm 4,8$ & $17 \pm 5,0$ & $18 \pm 7,1$ \\
\hline & G60 & $13 \pm 3,9$ & $13 \pm 2,1$ & $15 \pm 3,5$ & $17 \pm 5,0$ & $16 \pm 3,2$ \\
\hline & G40 & $14 \pm 5,4$ & $15 \pm 4,7$ & $13 \pm 4,4$ & $15 \pm 6,2$ & $14 \pm 4,5$ \\
\hline & G20 & $19 \pm 8,5$ & $19 \pm 6,4$ & $18 \pm 6,7$ & $20 \pm 10,0$ & $20 \pm 8,6$ \\
\hline \multirow{5}{*}{ PMCP } & G100 & $7 \pm 3,5$ & $6 \pm 4,7$ & $6 \pm 4,2$ & $7 \pm 4,4$ & $7 \pm 3,8$ \\
\hline & G80 & $8 \pm 3,9$ & $9 \pm 4,9$ & $9 \pm 4,0$ & $10 \pm 5,1$ & $11 \pm 7,3$ \\
\hline & G60 & $7 \pm 2,7$ & $6 \pm 2,7$ & $7 \pm 2,9$ & $9 \pm 3,6$ & $8 \pm 4,2$ \\
\hline & G40 & $8 \pm 6,3$ & $9 \pm 6,8$ & $6 \pm 1,7$ & $9 \pm 5,7$ & $8 \pm 3,0$ \\
\hline & G20 & $11 \pm 10,9$ & $10 \pm 7,3$ & $10 \pm 8,5$ & $12 \pm 11,4$ & $12 \pm 9,9$ \\
\hline \multirow{5}{*}{ RPT } & G100 & $4473 \pm 769$ & $4691 \pm 1038$ & $4232 \pm 1277$ & $4664 \pm 1458$ & $4286 \pm 1424$ \\
\hline & G80 & $4301 \pm 1963$ & $4137 \pm 1717$ & $4049 \pm 1432$ & $3919 \pm 1279$ & $3473 \pm 765$ \\
\hline & G60 & $4484 \pm 1750$ & $4472 \pm 1533$ & $4312 \pm 1380$ & $4616 \pm 1796$ & $4533 \pm 1659$ \\
\hline & G40 & $4383 \pm 1469$ & $4044 \pm 1395$ & $4418 \pm 1729$ & $4378 \pm 1564$ & $4000 \pm 1419$ \\
\hline & G20 & $4415 \pm 1416$ & $4111 \pm 1246$ & $3757 \pm 1016$ & $4546 \pm 1160$ & $4906 \pm 1696$ \\
\hline \multirow{5}{*}{ RVP } & G100 & $393 \pm 183$ & $404 \pm 114$ & $406 \pm 237$ & $400 \pm 238$ & $458 \pm 211$ \\
\hline & G80 & $385 \pm 182$ & $349 \pm 204$ & $349 \pm 209$ & $302 \pm 247$ & $328 \pm 190$ \\
\hline & G60 & $287 \pm 159$ & $304 \pm 149$ & $368 \pm 169$ & $362 \pm 227$ & $389 \pm 338$ \\
\hline & G40 & $303 \pm 185$ & $274 \pm 214$ & $322 \pm 144$ & $279 \pm 118$ & $252 \pm 99,46$ \\
\hline & G20 & $418 \pm 275$ & $395 \pm 281$ & $319 \pm 245$ & $314 \pm 262$ & $352 \pm 243$ \\
\hline
\end{tabular}

Médias seguidas por letras maiúsculas diferentes, nas colunas, diferem entre si pelo teste de Tukey $(\mathrm{P}<0,05)$. Médias seguidas por letras minúsculas diferentes, nas linhas, diferem entre si pelo teste de Tukey $(\mathrm{P}<0,05)$.

Ciência Rural, v.38, n.3, mai-jun, 2008. 
(ROBINSON, 1993). Portanto, mesmo com o emprego de diferentes concentrações de oxigênio, os valores registrados para PAMP estiveram dentro da normalidade (HASKINS, 1996), não havendo diferenças entre os grupos e dentro destes. KOJIMA et al. (2002), em seu estudo com cães, pré-medicados ou não, recebendo propofol sob respiração espontânea com ar ambiente, também não relataram diferenças nos valores de PAMP entre os valores basais e os registrados no decorrer do protocolo.

De acordo com GREENE et al. (1990) e MUIR III \& MASON (1996), a PMCP pode ser empregada como referência e representa boa estimativa da précarga, sendo que, nesse estudo, para tal parâmetro, não foram constatados interferências do emprego de diferentes $\mathrm{FiO}_{2}$.

As PMAP e PMCP mativeram-se no mesmo patamar em todos os grupos, durante todo o período experimental (Tabela 3), o que pressupõe estabilidade da função ventricular e pré-carga, já que esses parâmetros são rotineiramente empregados na clínica de emergência, para se estimar a pressão de enchimento do ventrículo esquerdo (MARK, 1998) e a pressão atrial esquerda, respectivamente (GREENE et al., 1990; MUIR III \& MASON, 1996; MARK, 1998). Dessa forma, a administração de diferentes $\mathrm{FiO}_{2}$ não foi capaz de causar alterações nessas variáveis, e, portanto, não prejudicou a estabilidade da função ventricular e pré-carga.

Relativamente ao DC, a variável não apresentou diferenças entre os grupos, bem como dentro destes (Tabela 1), não sendo observadas alterações decorrentes do emprego de diferentes concentrações de oxigênio em cães com respiração espontânea. Os mesmos resultados foram relatados por QUANTD et al. (1998) em cães submetidos à anestesia com dose única de propofol, com $\mathrm{FiO}_{2}$ a $100 \%$. Adicionalmente, é importante ressaltar que, embora o DC seja expresso matematicamente como o produto da FC pelo volume sistólico (VS) (MUIR III \& MASON, 1996), deve-se considerar outras variáveis que possam interferir diretamente sobre o parâmetro, principalmente atuando sobre o VS, o qual sabidamente sofre interferência direta da contratilidade cardíaca, da pré e pós-carga (GREENE et al., 1990). Dessa forma, a manutenção do DC durante todo o período experimental pode ser atribuída à estabilidade observada nas variáveis hemodinâmicas estudadas, que não sofreram interferências do emprego de diferentes $\mathrm{FiO}_{2}$, como descrito anteriormente.

A RPT não diferiu significativamente entre os grupos (Tabela 3). Considerando-se que a RPT é obtida através de calculo matemático (NUNES, 2002; VALVERDE et al., 1991), sendo que, neste estudo, os componentes da equação (PAM e DC) se mantiveram estáveis e não registraram alterações decorrentes do emprego de diferentes $\mathrm{FiO}_{2}$, fica clara a influência desses parâmetros sobre a variável. O mesmo ocorreu com relação à RVP, calculada a partir dos valores médios da PMAP, PMCP e DC (NUNES, 2002).

\section{CONCLUSÃO}

Conclui-se que, em cães submetidos à infusão contínua de propofol, na dose de $0,7 \mathrm{mg} \mathrm{kg}^{-1} \mathrm{~min}^{-1}$, e mantidos em ventilação espontânea, as variáveis hemodinâmicas não são afetadas pelo emprego de diferentes $\mathrm{FiO}_{2}$. Entretanto, ao avaliarem-se os dados hemogasométricos obtidos, é possível concluir-se que a melhor $\mathrm{FiO}_{2}$ a ser empregada no protocolo proposto é a de $60 \%$.

\section{AGRADECIMENTOS}

Os autores agradecem ao Conselho Nacional de Desenvolvimento Científico e Tecnológico (CNPq) e à Fundação de Amparo à Pesquisa do Estado de São Paulo (FAPESP), pelo apoio financeiro concedido, e ao Programa de Pós-graduação em Cirurgia Veterinária da Faculdade de Ciências Agrárias e Veterinárias - FCAV.

\section{FONTEDEAQUISIÇÃO}

${ }^{a}$ Diprivan, ZENECA Farmacêutica do Brasil Ltda., São Paulo, SP, Brasil.

${ }^{\mathrm{b}}$ OHMEDA, mod. Excel 210SE, Madison, EUA (Processo FAPESP 97/10668-4).

c Infusion Pump 670T, Samtronic, São Paulo, SP, Brasil.

${ }^{d}$ DIXTAL, mod. DX - 2010, Manaus, AM, Brasil (Processo FAPESP 96/02877-0 e 02/04625-0).

e Cateter Insyte - BD Insyte ${ }^{\circledR}$-Becton, Dickinson Indústria Cirúrgica, Juiz de Fora, MG, Brasil.

${ }^{\mathrm{f}}$ Hemogasômetro Roche Omi C - Rochi Diagnostics GmbH Mannheim, Alemanha.

${ }^{g}$ Cateter Swan-Ganz Pediátrico - 75 cm/5F (4 vias)- Edwards Lifesciences, Irvine, CA, EUA.

${ }^{\mathrm{h}}$ TEB- Mod. ECGPC software versão 1.10, São Paulo, SP, Brasil (Processo FAPESP 96/1151-5).

\section{COMITÊDEÉTICAEBIOSSEGURANÇA}

Este experimento foi aprovado pela Comissão de Ética e Bem-Estar Animal (CEBEA) da FCAV/Unesp, conforme o protocolo número 017678-06, sendo realizado de acordo com normas éticas. Terminado o estudo, os animais foram oferecidos para adoção.

\section{REFERÊNCIAS}

AKÇA, O. et al. Comparable postoperative pulmonary atelectasis in patients given $30 \%$ or $80 \%$ oxygen during and 2 hours after colon resection. Anesthesiology, v.91, n.4, p.991998, 1999.

Ciência Rural, v.38, n.3, mai-jun, 2008. 
BUFALARI, A. et al. Comparative responses to propofol anesthesia alone with alpha 2-adrenergic medications in a canine model. Acta Vet Scand, v.37, n.2, p.187-201, 1996.

CLAYBAUGH, J.R. et al. Effects of $\mathrm{FiO}_{2}$ on $\mathrm{O}_{2}$ consumption and cardiovascular and hormonal responses to hemorrhage in the goat. Mil Med, v.168, n.9, p.758-764, 2003.

DETWEILER, D.K. Mecanismo de controle do sistema circulatório. In: SWENSON, M.J.; REECE, W.O. Dukes: fisiologia dos animais domésticos. 11.ed. Rio de Janeiro: Guanabara Koogan, 1993. Cap.10, p.170-208.

DUKE, T. A new intravenous anesthetic agent: propofol. Can Vet J, v.36, p.181-183, 1995.

FERRO, P.C. et al. Variáveis fisiológicas em cães submetidos à infusão contínua de diferentes doses de propofol. Ciênc Rural, Santa Maria, v.35, n.5, p.1103-1108, 2005.

FUJII, Y. et al. The dose-range effects of propofol on the contractility of fatigued diaphragm in dogs. Anesth Analg, v.93, p.1194-1198, 2001.

GREENE, S.A. et al. Cardiovascular effects of butorphanol in halothane-anesthetized dogs. Am J Vet Res, v.51, n.8, p.12761279, 1990.

GUYTON, A.C. Regulação nervosa da circulação e controle rápido da pressão arterial. In: GUYTON. A.C. Tratado de fisiologia médica. 8.ed. Rio de Janeiro: Interamericana, 1992. Cap.18, p.170-179.

HASKINS, S.C. Monitoring the anesthetized patient. In THURMON, J.C. et al. Lumb \& Jones' veterinary anesthesia. 3.ed. Philadelphia: Lippincott Williams \& Wilkins, 1996. Cap.1, p.3.

HOUPT, T.R. Equilíbrio ácido-básico. In: SWENSON, M.J.; REECE, W.O. Dukes: fisiologia dos animais domésticos. 11.ed. Rio de Janeiro: Guanabara Koogan, 1993. Cap.32, p.549-559.

JEFFERIES, A.R. Pathology. In: HALL, L.W.; TAYLOR, P.M. Anaesthesia of the cat. London: Baillière Tindall, 1994. Cap.4, p.63-88.

KIM, S.H. et al. Hypothermia, but not $100 \%$ oxygen breathing, prolongs survival time during lethal uncontrolled hemorrhagic shock in rats. J Trauma, v.44, n.3, p.485-491, 1998.

KOJIMA, K. et al. Effects of medetomidine-midazolam, acepromazine-butorphanol e midazolam-butorphanol on induction dose of thiopental and propofol and on cardiopulmonary changes in dogs. Am J Vet Res, v.63, n.12, p.1671-1679, 2002.

MAGNUSSON, L.; SPAHN, D.R. New concepts of atelectasis during general anaesthesia. Br J Anaesth, v.91, n.1, p.61-72, 2003.

MARK, J.B. Atlas of cardiovascular monitoring. New York: Churchill Livingstone, 1998. 362p.

MORGAN, D.W.T.; LEGGE K. Clinical evaluation of propofol as an intravenous anesthetic agent in cats and dogs. Vet Rec, n.1, p.31-33, 1989 .
MUIR III, W.W.; MASON, D. Cardiovascular system. In: THURMON, J.C. et al. Lumb \& Jones' veterinary anesthesia. 3.ed. Philadelphia: Lippincott Williams \& Wilkins, 1996. Cap.5, p.62-114.

MUIR III, W.W.; HUBBELL, J.A.E. Manual de anestesia veterinária. 2.ed. Porto Alegre: Artmed, 1997. 503p.

NUNES, N. Monitoração da anestesia. In: FANTONI, D.T.; CORTOPASSI, S.R.G. Anestesia em cães e gatos. São Paulo: Roca, 2002. Cap.6, p.64-81.

O'FLAHERTY, D.et al. Capnography - principles and practice series. London: BMJ, 1994. 108p.

QUANDT, J.E. et al. Cardiorespiratory and anesthetic of propofol and thiopental in dogs. Am J Vet Res, v.59, n.9, p.1137-1143, 1998.

ROBINSON, N.E. Fluxo sangüíneo pulmonar. In: CUNNINGHAM, J.G. Tratado de fisiologia veterinária. Rio de Janeiro: Guanabara Koogan, 1993. Cap.44, p.380-385.

ROMALDINI, H. Repercussões cardiovasculares da ventilação mecânica. In: AULER JR, J.O.C.; AMARAL, R.V.G. Assitência ventilatória mecânica. São Paulo: Atheneu, 1995. Cap.8, p.115-119.

ROTHEN, H.U. et al. Atelectasis and pulmonary shunting during induction of general anaesthesia - can they be avoided? Acta Anaesthesiol Scand, v.40,n.5, p.524-529, 1996.

SENIOR, D.F. Fluidoterapia, eletrólitos e controle ácido-básico. In: ETTINGER, S.J.; FELDMAN, E.D. Tratado de medicina interna veterinária. 4.ed. São Paulo: Manole, 1997. Cap.60, p.420-445.

SISSON, D. Avaliação clínica da função cardíaca. In: ETTINGER. S.J. Tratado de medicina interna veterinária. 3.ed. São Paulo: Manole, 1992. p.670-686.

STRANDBERG, A. et al. Atelectasis during anaesthesia and in the postoperative period. Acta Anaesth Scand, v.30, p.154158, 1986

TILLEY, L.P. Essentials of canine and feline electrocardiographic: interpretation and treatment. 3.ed. Philadelphia: Lea \& Feabinger, 1992. 470p.

VALVERDE, A. et al. Comparison of the hemodynamic effects of halothane alone and halothane combined with epidural administered morphine for anesthesia in ventilated dogs. Am $\mathbf{J}$ Vet Res, v.52, n.3, p.505-509, 1991.

WATKINS, S.B. et al. Propofol as an intravenous anaesthetic agent in dogs. Vet Rec, v.120, p.326-329, 1987.

WHITE, F.P. Propofol. In: WHITE, P.F. Tratado de anestesia venosa. Porto Alegre: Artmed, 2001. p.121-175.

ZAPPELLINI, A. et al. Evaluation of two different oxygen inspiratory fractions on the hemodynamic effects of $\mathrm{N}$ omeganitro-Larginine methyl ester in anesthetized dogs. Braz J Med Biol Res, v.29, n.1, p.33-39, 1996. 